\title{
Large Format Encapsulation
}

\author{
Eric Kuah, Hao Ji Yuan, Yuan Bin, Chan Wei Ling, Wu Kai and Ho Shu Chuen \\ ASM Technology Singapore Pte Ltd., 2 Yishun Avenue 7, Singapore 768924
}

\begin{abstract}
This paper will review the challenges on large format encapsulation with respect to the control of mold cap thickness, and the encapsulant impact on moldability such as flow mark on final product. Control of mold co-planarity is best performed dynamically during molding, otherwise it would be challenging to obtain good co-planarity within $20 \mu \mathrm{m}$. Moldability demand such as encapsulant coverage with highly viscous material, flowability and flow mark will be discussed. Warpage control depends heavily on the formulation of the encapsulant and the form of encapsulant, i.e., granular/powder, liquid and sheet.
\end{abstract}

Key words: Co-planarity, dynamic control, encapsulant, flowability, flow mark and warpage.

\section{Introduction}

The semiconductor industry has undergone dramatic changes for several decades from MM (More Moore) to MTM (More Than Moore) and now to SM (System Moore) [1]. The era of MM focused on bulky equipment such as work stations, desktop personal computing and super computers, while MTM is about portability like cellphones then and today's smartphones and tablets. Now SM concentrates on wearables, Internet of Things (IoT) and Internet of Everything (IoE). The implications of these trends are miniaturization, all-in-one PEMs (plastic encapsulated microelectronics) and high functional density.

In line with this development the assembly and packaging for the back-end (PABE) segment of the semiconductor industry are also trending their design towards miniaturization, compact form factor packages, multifunctional/multiple silicon dies, MUF/CUF and I/O (input-output connections) that can exceed 1,000 which is unheard of a decade ago for PEMs. As an upshot of these new demands, packaging, process and equipment engineers have to innovate and develop solutions to meet such challenges.

There are many reasons for designers and

Corresponding author: Eric Kuah, DBA, vice president of technology, encapsulation solutions group, ASMPT, research fields: heat transfer, fluid mechanics, FEM, CFD and encapsulation process development for advanced packaging. manufacturers to trend towards large format substrate for PEMs packaging like value-added packaging, extreme form factor reduction, flexibility and cost among others [2-4]. Value-added packaging includes the move towards more than Moore's Law at panel system level, and additional special semi-conductor features such as anti-voltage spiking, extreme low capacitance etc. [2]. We had experienced in our course of work that to achieve extreme form factor, end users leveraged on large panel to produce millions of parts per encapsulation cycle of the size of $100 \mu \mathrm{m} \times 200 \mu \mathrm{m}$ $\times 75 \mu \mathrm{m}$ after saw singulation which is impossible to realize with traditional leaded or non-leaded substrates. The advance in large format packaging is mainly driven by consumer electronics, mobile communications and the up and coming IoT.

In large form factor encapsulation, an end user can derive the effect of what we have termed as CCCV, where the components of performance, value and cost can be clubbed together. The acronym, CCCV stands for Cost-effective design, Cost-effective solution and Cost-to-deliver value for customers. In the encapsulation industry which we are part of, we are witnessing this CCCV syndrome, where there are constant enquiries by end users who are thinking of switching from the standard strand of substrate width with several rows and columns of their PEMs to large format in order to have a large gain of units produced 
during a typical encapsulating cycle. In this paper we will discuss some of the challenges of large format encapsulation from both the perspective of controlling the mold cap thickness, moldability and warpage and the angle of encapsulant.

\section{Mechatronics Press for Co-planarity Control}

Co-planarity control, which is actually mold cap thickness variation, becomes critical in large format encapsulation as we drive the encapsulated thickness down to below $100 \mu \mathrm{m}$. For instance we cannot accept a mold cap thickness of $70 \mu \mathrm{m}$, when a typical mold cap thickness is $\pm 30 \mu \mathrm{m}$, as the variation is huge. To cater to such large variation, we had in our course of work designed a mechatronics press, which we termed it as the "Pillar-less Press: PLP", which will dynamically control the final mold cap thickness to within $20 \mu \mathrm{m}$.
Fig. 1 shows the comparison of how PLP and mechanical press work to dynamically control the final mold cap thickness during an encapsulation cycle. This PLP has two columns of solid side wall that are not found in a traditional mechanical molding press. Other key features of this PLP include a cam and motorized mechanism to drive the platen towards an optimal position so that the mold cap tolerance can be tightly adjusted and controlled during the encapsulation process. Electronics sensors with advanced feedback control algorithm are used to monitor and feedback the co-ordinate location for optimal final encapsulation before packing prior to final encapsulant cure. Fig. 2 shows the result of mold cap thickness tolerance that can be achieved using PLP to mold a $\Phi 12$-inch $750 \mu \mathrm{m}$ thick wafer to a mold cap thickness of $95 \mu \mathrm{m}$.

The result showed that for a mold cap thickness of $95 \mu \mathrm{m}$, using PLP we can get a thickness range between $91 \mu \mathrm{m}$ and $98 \mu \mathrm{m}$, a difference within a range

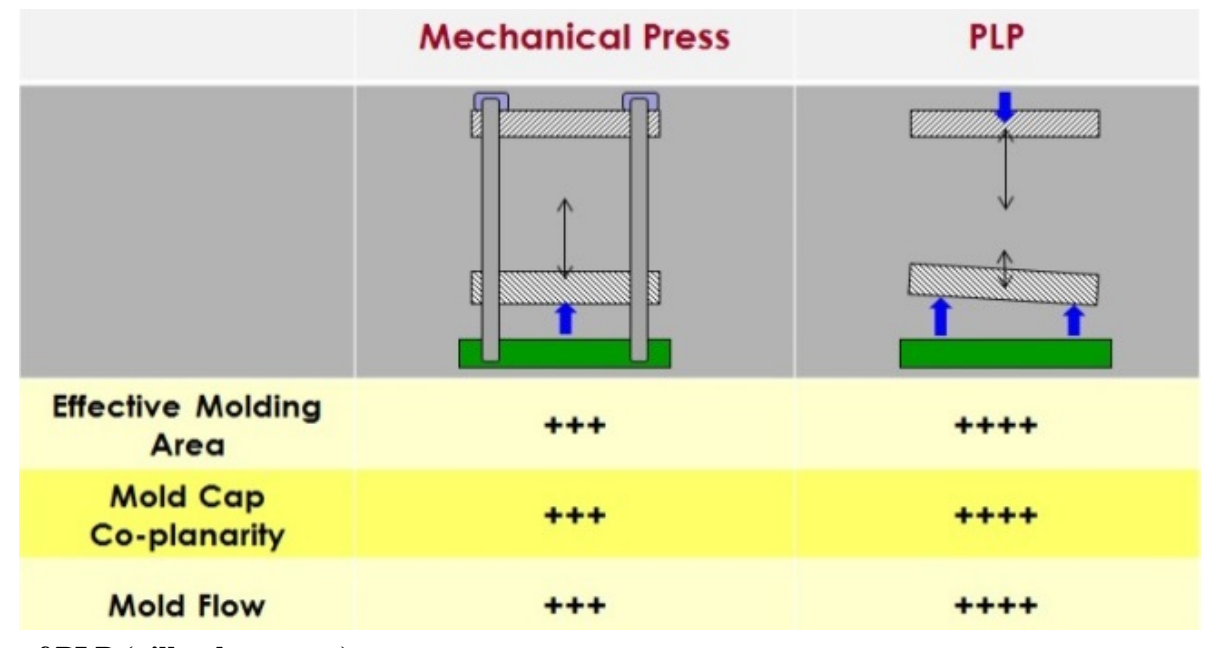

Fig. 1 Schematic of PLP (pillar-less press).
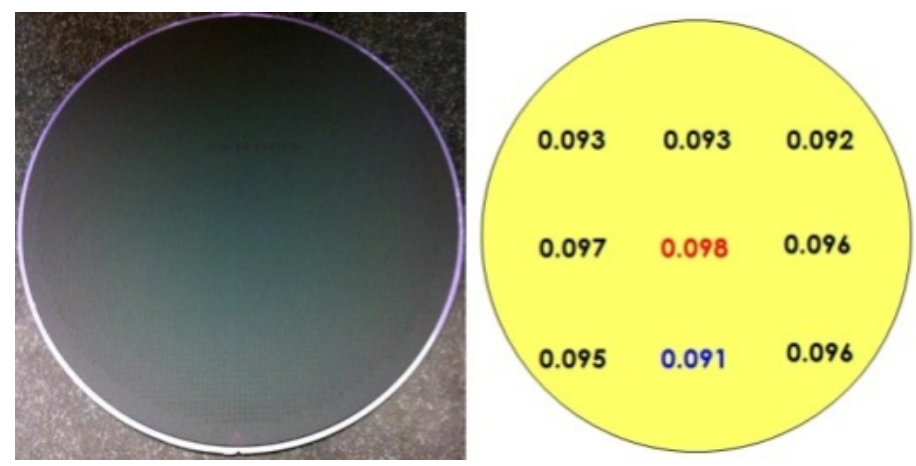

Units: $\mathbf{m m}$

Average: 0.095

Minimum: 0.091

Maximum: 0.098

Range: 0.007

Fig. 2 Mold cap thickness of $95 \mu \mathrm{m}$. 
of $7 \mu \mathrm{m}$ and an SD of $2.5 \mu \mathrm{m}$. This indicated that the advanced dynamic control of PLP is able to meet the design specification of mold cap tolerance of $\pm 20 \mu \mathrm{m}$.

Two additional confirmation tests of the PLP design were performed to validate self-compensation capabilities. The first test was to use a metal carrier that had been deliberately fabricated with an uneven surface to evaluate if PLP has the capability to compensate the mold cap thickness. Figs. 3 and 4 are the results of this evaluation.

Fig. 3 shows the comparison between mold cap tolerance when PLP advanced control is deactivated and activated. The results indicated that it is not possible to achieve a co-planarity tolerance of $20 \mu \mathrm{m}$ if the PLP advanced thickness control is not activated. Fig. 4 is the result of the repeatability test carried out to validate that the finding in Fig. 3 is not a one-off observation. Additional 20 more sets of encapsulation trials were conducted with PLP advanced control activated on different carrier slant thickness. The results are shown in Fig. 4.

The result from these 20 mold trials clearly showed that PLP designs helped to control the mold cap thickness to within specifications, supporting the fact that for large form factor encapsulation it is critical to have such dynamic compensation of mold cap thickness in place.

The last test of the dynamic compensation on PLP evaluated its performance in the event if the dispensed encapsulant has an uneven distribution (off-set) either during dispensing or transporting to the molding press. The results of this evaluation are shown in Figs. 5 and 6. The results in Fig. 5 supported our observation that if the PLP dynamic compensation is turned off the co-planarity tolerance will be out of specification. The outcome becomes positive when the PLP dynamic compensation is activated, meeting the design co-planarity specification. Fig. 6 is the result derived

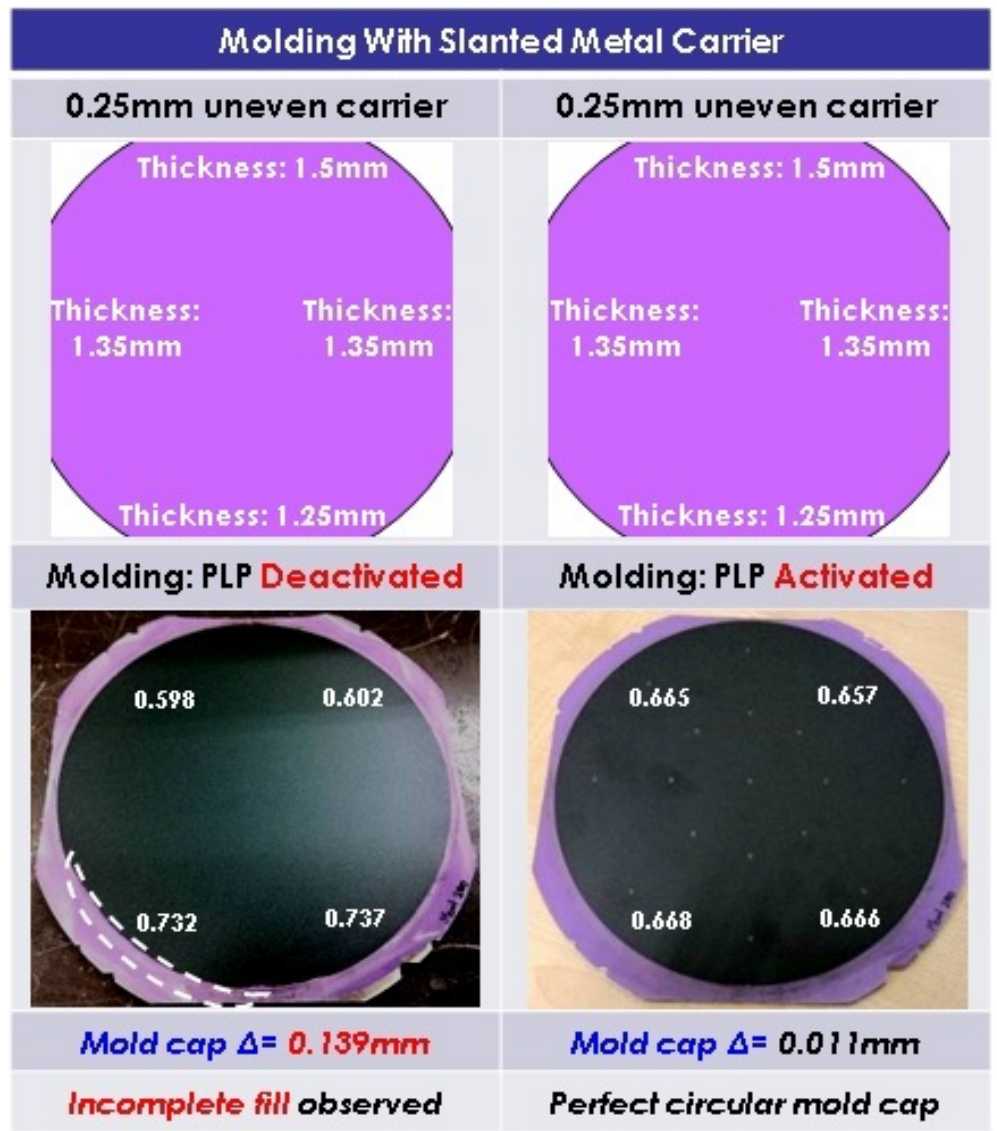

Fig. 3 Results of slanted metal carrier. 


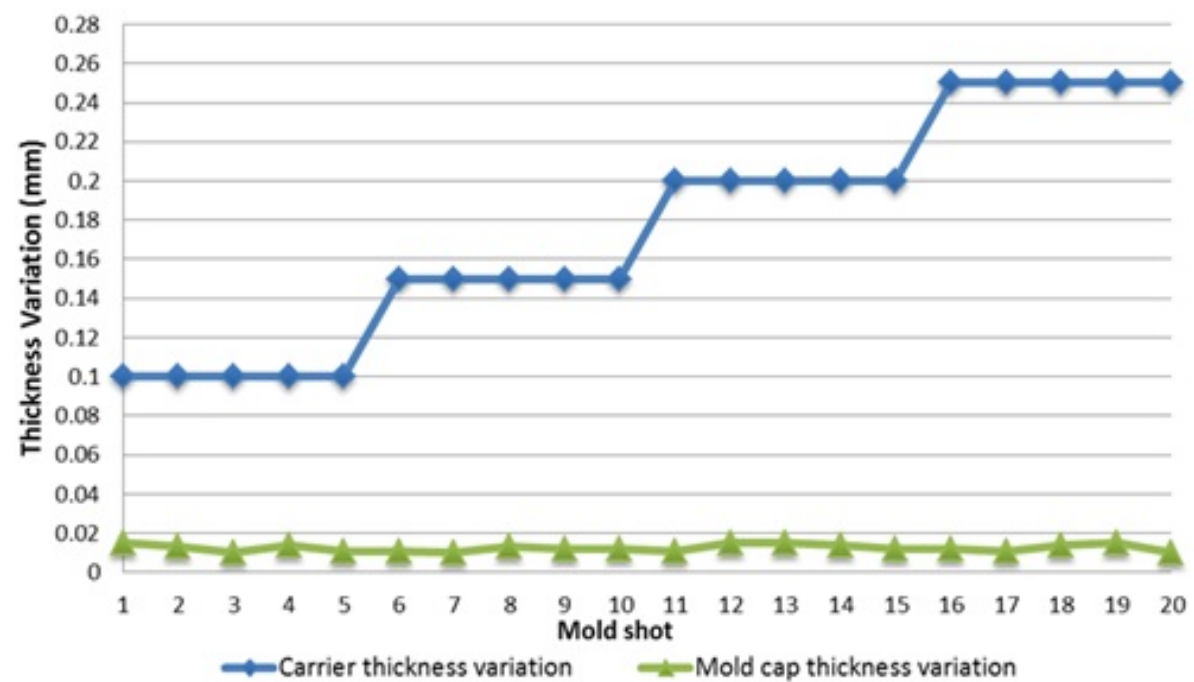

Fig. 4 Results of different carrier thickness variations.

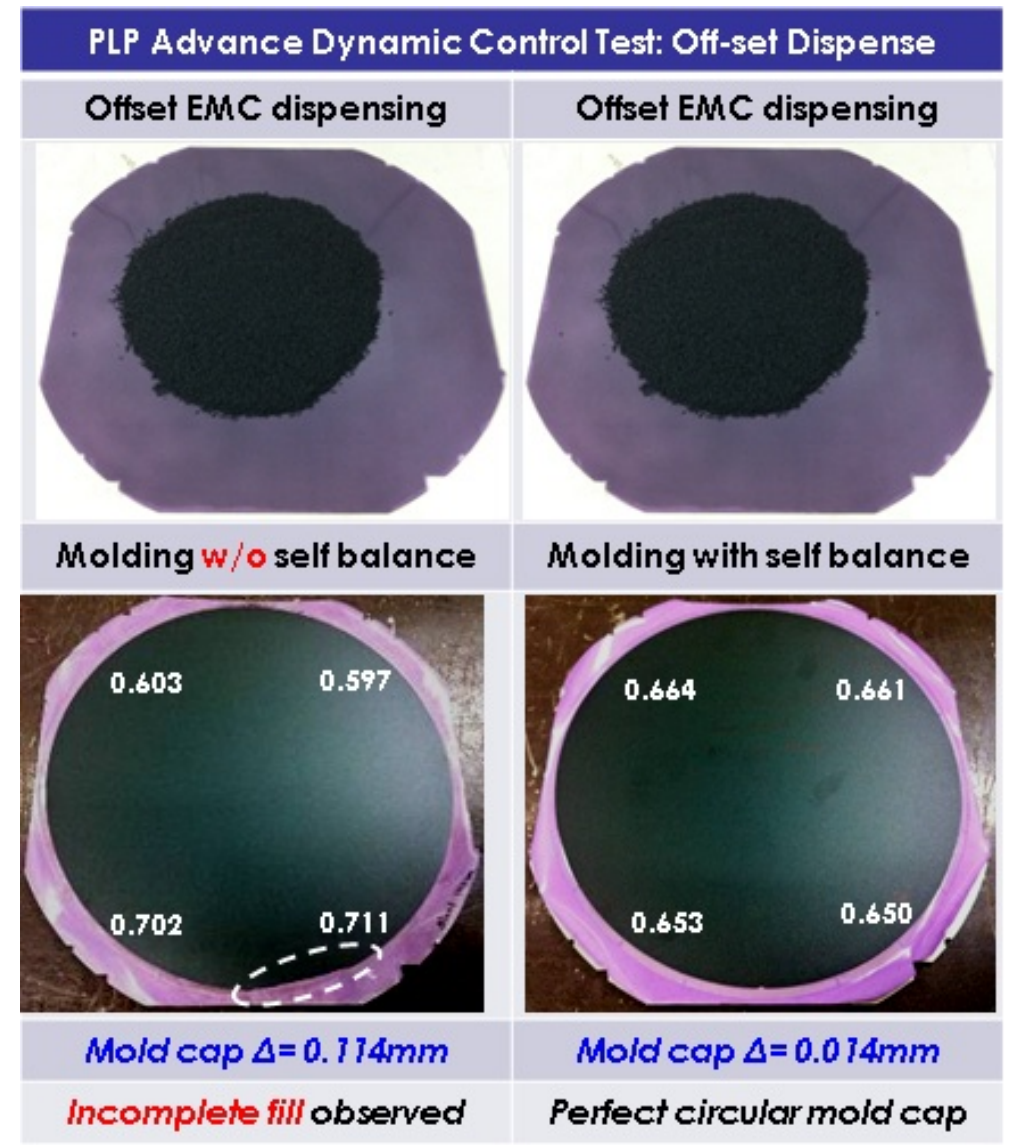

Fig. 5 Mold cap thickness for off-set dispensing.

which confirmed that dynamic compensation is required to achieve within the co-planarity specification if the dispensed encapsulant becomes off-set due to the reasons stated above.

The results shared so far indicated that the advanced dynamic compensation control design of PLP has the ability to deliver a controlled tight mold cap thickness even if the substrate thickness varies, or the dispensed encapsulant becomes unevenly distributed due to dispensing and/or handling shortcoming. 


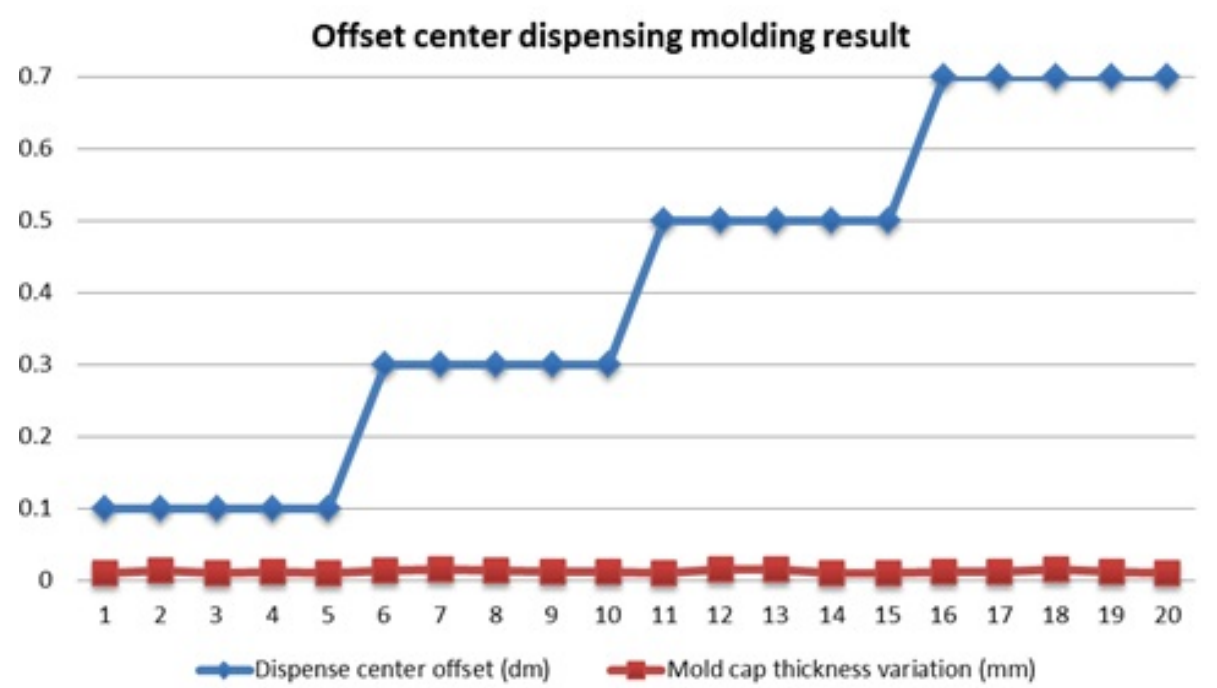

Fig. 6 Mold cap thickness at different off-set dispensing.

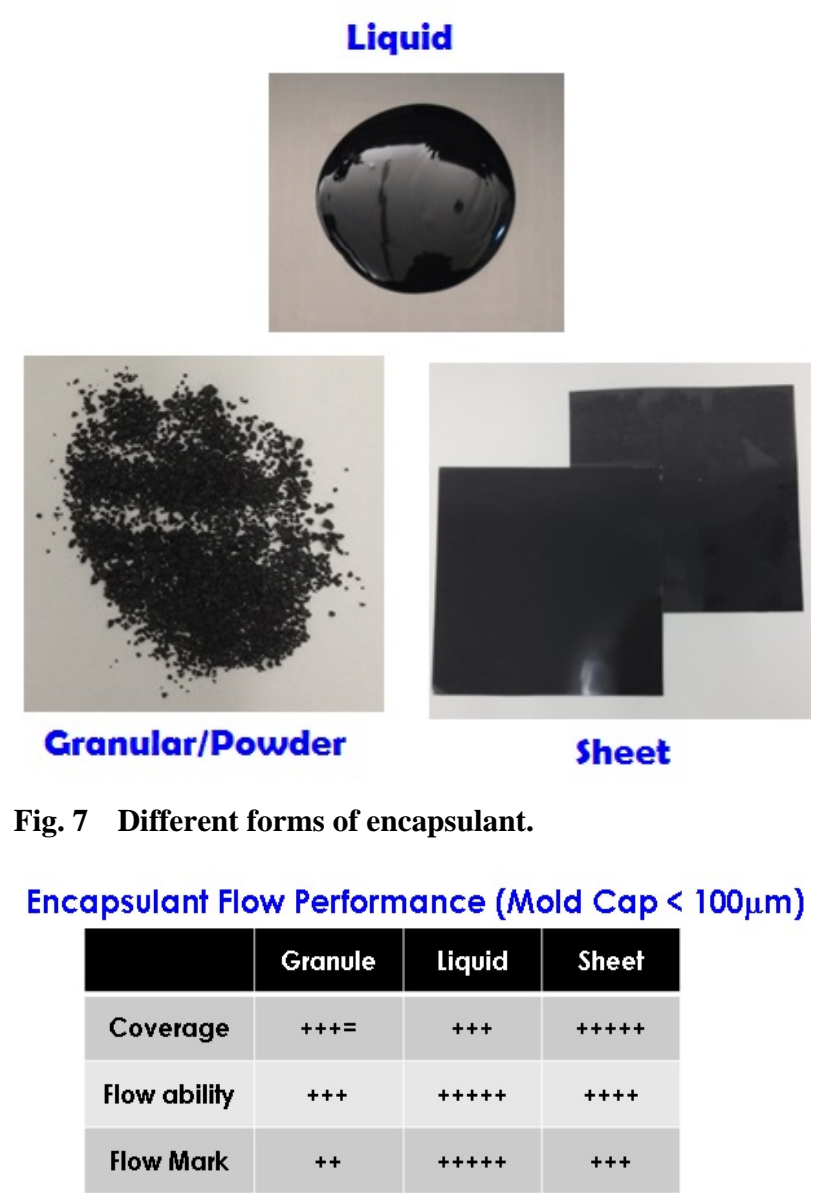

Fig. 8 Encapsulant flow performance.

\section{Moldability Is Impacted by Encapsulant}

The three forms of encapsulant that can be used for large format encapsulation are liquid, granular/powder and molding sheet. Please refer to Fig. 7. Each form of encapsulant has its advantages and disadvantages and outcome will largely depend on package requirements such as reliability, functionality, packaging platform, moldability, and cost benefits. A comparative study was performed on these three forms of encapsulant to evaluate their coverage (scalability), flowability and methods of molding, i.e., die up or die down. In terms of molding coverage the molding sheet ranked top. This is because the MS (molding sheet) when placed over the areas of semiconductor chips acts as a protection while liquid and granular/powder had to be dispensed. As a result MS can be tailored to cover a larger surface area of the silicon chip. Liquid ranked lower than granular/powder in term of coverage once the liquid viscosity becomes greater than $700 \mathrm{~Pa}-\mathrm{s}$. To dispense such viscous liquid encapsulant, special techniques are required like pre-heating of encapsulant over the dispensed target area at a controlled rate so as to avoid the kick-starting the chemical curing of the liquid encapsulant.

Fig. 8 is a comparison of performance for these three forms of encapsulant. These three forms of encapsulant had been used for molding thin mold cap. Their typical viscosity liquid, powder and MS are 250 Pa-s, 160 Pa-s and $\sim 30$ Pa-s respectively. The flowability for liquid is the best because it is in a semi-solid state 
compared to the solid granular/powder and molding sheet which has B-stage material. Granular/powder ranked the lowest ranking for flowability among the three and it can also lead to flow mark related challenges. Some possible root causes of such molding defect include filler size to mold cap ratio and flow distance from the dispensed area to the edge of wafer. The viscosity liquid and MS are at the extreme, resulting in different flow mark performance. In addition, the challenge of having good flowability with no flow mark on the surface of the encapsulated large format package is compounded by the fact that these samples are molded at less than $100 \mu \mathrm{m}$ thickness.

Figs. 9-11 illustrated the experimental observation of flow mark for different forms of encapsulant. Liquid encapsulated product does not have blemish at the edge compared to granular/powder and sheet. This demonstrated that the viscosity of an encapsulant (typical viscosity liquid, powder and MS are 250 Pa-s, $\sim 160$ Pa-s and $\sim 30$ Pa-s respectively) does not impact flowability and flow mark performance when low viscosity material supposedly should have a better flow under heat. One plausible explanation would be the chemistry used in formulating the encapsulant and the form that it was supplied. Another conceivable reason that we could think of is non-optimal processing conditions during molding. This turned out to be a non-issue, as many attempts had been made during the course of this work to optimize the molding process conditions but similar flow mark outcome was obtained.

\section{Warpage Performance of Large Format Package}

Warpage is defined an out-plane bending and deformation [5]. There are many factors that influence warpage such as composition of encapsulant, molding temperature, package form factor/geometry and CTE mismatch to name a few. Large format package tends to warp more severely as it involves packaging a large surface area coupled with slimness and brittleness of the substrate used [5]. In this section we will discuss the result of the three types of encapsulant (namely liquid, granular/powder and molding sheet) and also the impact on features within the substrate. If special and entrenched features are built into the substrate that is below the $\mathrm{X}-\mathrm{Y}$ plane it tends to warp more when compared to the encapsulant that is above the $\mathrm{X}-\mathrm{Y}$ plane. The reason is because entrenched features will change the mechanics of shrinkage as they will weaken the flexural strength of substrate which easily "yields" to compound shrinkage. This is compounded by the amount of encapsulant required to burry these entrenched feature to protect the circuitry. Fig. 12 shows

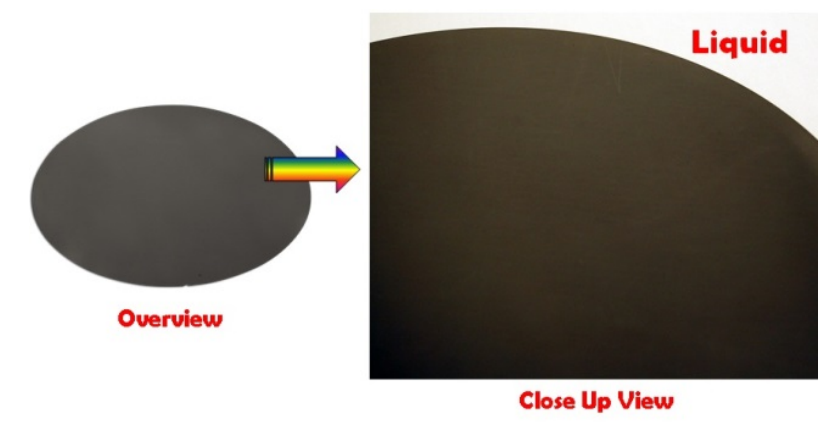

Fig. 9 Flow mark of liquid encapsulant.

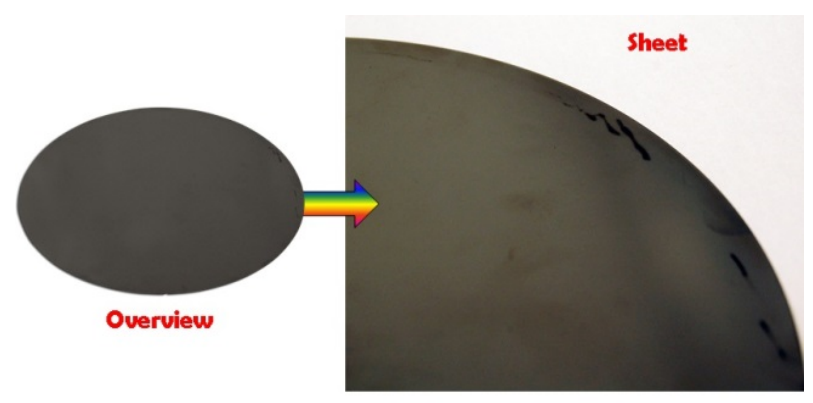

Close Up View

Fig. 10 Flow mark of molding sheet.

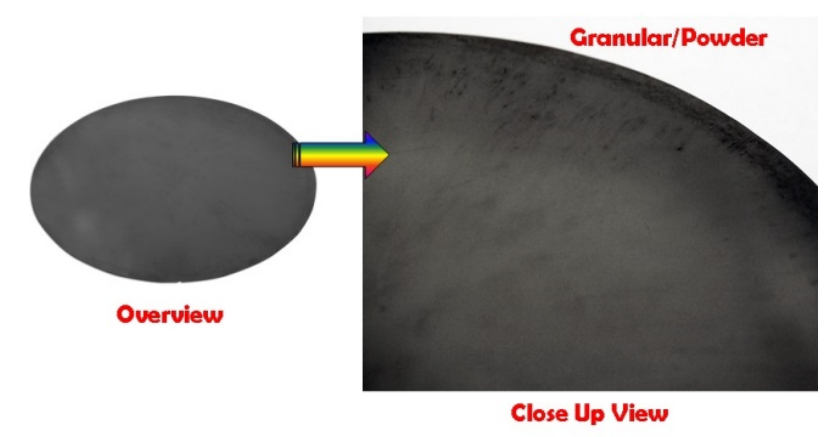

Fig. 11 Flow mark of granular/powder encapsulant. 

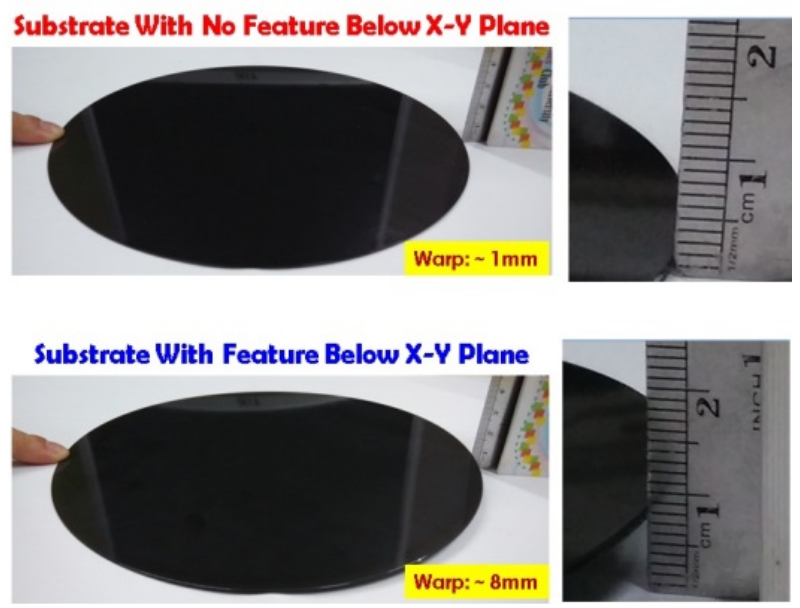

Fig. 12 Warpage comparison feature vs. no feature.

\section{Substrate With No Feature Below X-Y Plane}

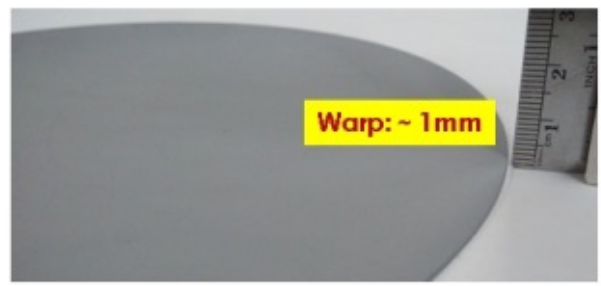

Substrate With Feature Below X-Y Plane

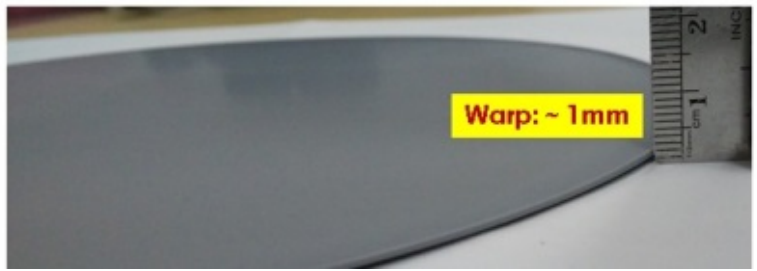

Fig. 13 Well design encapsulant can result in good warpage for substrate with either feature or no feature.

Table 1 Warpage after molding and PMC for $<100 \mu \mathrm{m}$ mold cap thickness.

\begin{tabular}{lll}
\hline \multirow{2}{*}{ Type of encapsulant } & $\begin{array}{l}\text { Warpage after } \\
\text { molding }(\mathrm{mm})\end{array}$ & $\begin{array}{l}\text { Warpage after } \\
\text { PMC }(\mathrm{mm})\end{array}$ \\
\hline Liquid & $<1$ & $<1$ \\
Granular/powder & $\sim 3.5$ & $\sim 3$ \\
Sheet & $\sim 7$ & $\sim 5$ \\
\hline
\end{tabular}

that there would be a difference when the quantity of encapsulant used on large format substrate molding varied even if liquid encapsulant and molding conditions remain the same and the final mold cap thickness is maintained above the $\mathrm{X}-\mathrm{Y}$ plane. From this result, it can be said that when designing a package using a large format substrate one should perform due diligence prior to designing the encapsulant by feeding back as much relevant information as possible to the encapsulant manufacturer. Otherwise, it would take considerable time to arrive at the right material design. Fig. 13 shows that it is possible to have good warpage using the same material regardless whether it has a feature or no feature on the large format substrate.

Table 1 compares the warpage performance of the various encapsulants used in the work after mold and PMC (post mold curing). Based on a number of experiments carried out using different chemistry computation of encapsulant, it is found that liquid encapsulant offered the best warpage performance. The liquid used in large format typically has low CTE material, which in turn leads to lower warpage due to less shrinkage when the temperature changed from molding to ambient. Comparing the modulus of compound with the substrate used, the compound is low but has a high CTE compared to silicone (CTE 2.6 $\mathrm{ppm})$. The resultant warpage is low because encapsulant modulus is much lower than silicone (Young Modulus $131 \mathrm{GPa}$ ).

Most material shrinks during molding, but some do expand and will lead to a lower warpage, which is the result of the interaction between CTE and modulus. PMC can be used to reduce warpage level. However, the acceptance of this reduction in warpage level will depend on whether the post encapsulation process is able to handle the molded package for further assembly processing such as grinding, singulation etc.

\section{Summary of Work}

This work is about the challenges that a packaging engineer would face when encapsulating a large format package. The term large format is used here because the data that are shared above used a round wafer which can also be applied on a large square or rectangle substrate. The mechatronics press design, which we termed it as the "Pillar-less Press: PLP" can dynamically control the final mold cap (co-planarity) to within $20 \mu \mathrm{m}$ during the encapsulation process. PLP 
is able to perform two additional functions: (1) it can compensate for uneven substrate thickness and (2) uneven dispensed encapsulant to achieve within specification mold cap thickness tolerance. Evaluation of three forms of encapsulant for less than $100 \mu \mathrm{m}$ mold cap thickness is also discussed. For large coverage (scalability), an encapsulant in form of molding sheet will be the best among the three, however, it does not give perfect flowability and flow mark. The worst performer for flowability and flow mark is granular/powder. Liquid encapsulant offers the best performance for flowability, flow mark and warpage.

\section{Acknowledgements}

We wish to acknowledge and thank our colleagues of ASMPT, our customers (past and current) and material vendors (encapsulant and film) who had worked with us on the various large format encapsulation projects which led to this sharing of knowledge. No names are mentioned so as to protect the identity of the various parties.

\section{References}

[1] Rao, R. T. 2015. System Scaling: Glass Panel for System Components \& Si Wafer for Device Components. MiNaPad, Grenoble, France, 1-58.

[2] Daijiro, I., Shinya, S., Yoshikatsu, I., Shinya, I. et al. May 2015. "Integrated Module Structure of Fan-out Wafer Level Package for Terahertz Antenna.” In Proceeding of 65th Electronic Components \& Technology Conference (ECTC 2015), 1085-9.

[3] Meyer, T., Ofner, G., Bradl, S., Brunnbauer, M., and Hagen, R. Dec. 2008. "Embedded Wafer Level Ball Grid Array (eWLB).” In Proceeding 2008 10th Electron Packaging Technology Conference (EPTC 2008), Singapore, 994-8.

[4] Braun, T., Raatz, S., Voges, S., Kahle, R. et al. May 2015. "Large Area Compression Molding for Fan-out Panel Level Packing." In Proceeding of 65th Electronic Components \& Technology Conference (ECTC 2015), 1077-83.

[5] Ardebili, H., and Pecht, M. G. 2009. Encapsulation Technologies for Electronics Application (1st ed.). Elsevier Inc., United States of America, Chapter 5, 242-5. 